\title{
Academy of Medicine-Ministry of Health Clinical Practice Guidelines: Assessment and Management of Infertility at Primary Healthcare Level
}

Seong Feei Loh, Rachna Agarwal, Jerry Chan, Sing Joo Chia, Li Wei Cho, Lean Huat Lim, Matthew Sie Kuei Lau, Sheila Kia Ee Loh, Marianne Sybille Hendricks, Suresh Nair, Joanne Hui Min Quah, Heng Hao Tan, PC Wong,

ABSTRACT The Academy of Medicine (AMS) and Ministry of Health (MOH) have developed the clinical practice guidelines on Assessment and Management of Infertility at Primary Healthcare Level to provide doctors and patients in Singapore with evidence-based treatment for infertility. This article reproduces the introduction and executive summary (with recommendations from the guidelines) from the AMS-MOH clinical practice guidelines on Assessment and Management of Infertility at Primary Healthcare Level, for the information of SMJ readers. Chapters and page numbers mentioned in the reproduced extract refer to the full text of the guidelines, which are available from the Ministry of Health website: http://www.moh.gov.sg/content/moh_web/healthprofessionalsportal/doctors/guidelines/cpg_ medical/2013/cpgmed_infertility.html. The recommendations should be used with reference to the full text of the guidelines. Following this article are multiple choice questions based on the full text of the guidelines.

\subsection{Objectives and scope of guideline}

These guidelines are intended to assist primary care physicians and other healthcare professionals in the management of infertility. This includes increasing awareness and educating about the scale of fertility problems, recognising the various causes of infertility, natural fecundity, and success rates of in vitro fertilisation (IVF) treatment in relation to female age. These guidelines also seek to establish clear referral criteria to guide primary care physicians.

\subsection{Target group}

The primary target group of these guidelines are primary care physicians. Nevertheless, these guidelines would also benefit all healthcare professionals involved in the management of infertility.

\subsection{Guideline development}

These guidelines have been produced by a committee appointed by the Ministry of Health and the Academy of Medicine, Singapore, with representation from gynaecologists, fertility specialists, urologists and family physicians. They were developed using the best available current evidence and expert opinion.

\subsection{Review of guidelines}

Evidence-based clinical practice guidelines are only as current as the evidence that supports them. Users must keep in mind that new evidence could supersede recommendations in these guidelines. The workgroup advises that these guidelines be scheduled for review three years after publication, or if new evidence appears that requires substantive changes to the recommendations.

\section{EXECUTIVE SUMMARY OF RECOMMENDATIONS}

Details of the recommendations listed can be found in the main text as the pages indicated.

\section{Definition and causes of infertility}

GPP People who have not conceived after one year of regular unprotected sexual intercourse should be offered further clinical investigation, including semen analysis and/or assessment of ovulation (pg 20).

GPP

\section{Basic measures to optimise fertility potential}

D Preconceptual counselling in those with advanced age should include a discussion of the increased risks of aneuploidy, spontaneous abortion and obstetric complications (such as delivery by Caesarean section and gestational diabetes) associated with increasing maternal age (pg 21).

Grade D, Level 3

D In women with advanced maternal age (> 35 years), consultation with a reproductive specialist should be considered after six months of unsuccessful efforts to conceive (pg 22).

Grade D, Level 4 
D Sexual intercourse every 2-3 days is recommended to optimise the chance of pregnancy; this is less stressful than timing intercourse to coincide with ovulation, which is not recommended unless in circumstances preventing regular intercourse (pg 22).

Grade D, Level 4

B Women trying to get pregnant should be advised against excessive alcohol consumption of more than two drinks a day and episodes of binge drinking can cause fetal harm (pg 23).

Grade B, Level 2++

C Men should be warned that excessive alcohol intake is detrimental to semen quality (pg 23).

Grade C, Level 2+

B Women should be informed that smoking is likely to reduce their fertility (pg 23).

Grade B, Level 1+

D Men who smoke should be informed that smoking is associated with reduced sperm parameters (pg 24).

Grade D, Level 4

B Women trying to achieve a pregnancy should be informed that a body mass index (BMI) of $19-29 \mathrm{~kg} / \mathrm{m}^{2}$ is optimal (pg 24).

Grade B, Level 1+

B Couples seeking treatment for infertility should be routinely screened for usage of long-term prescription medication, as some have been known to affect fertility (pg 25).

Grade B, Level 2++

C Couples seeking treatment for infertility should also be routinely screened for occupational hazards and given appropriate advice (pg 25).

Grade C, Level 2+

A Women intending to become pregnant should be informed that dietary supplementation with folic acid before conception and up to 12 weeks' gestation reduces the risk of having a baby with neural tube defects. The recommended dose is $0.4 \mathrm{mg}$ per day. For women who have previously had an infant with a neural tube defect or who are receiving anti-epileptic medication, a higher dose of $5 \mathrm{mg}$ per day is recommended (pg 25).

Grade A, Level 1++

GPP Women who are concerned about their fertility should be offered rubella susceptibility screening so that those who are susceptible to rubella can be offered rubella vaccination and be advised not to become pregnant for at least one month following vaccination (pg 26).

GPP

GPP To avoid delay in fertility treatment, a specific enquiry about the timing and result of the most recent cervical smear test should be made to women who are concerned about their fertility. Cervical screening should be offered in accordance with the national cervical screening programme guidance (pg 26).

GPP

\section{Assessment and basic investigations of infertility (including referral)}

D Couples with fertility concerns should be interviewed separately as well as together, to bring out important history that a partner may want confidential from the other (pg 27).

Grade D, Level 4

D At the initial consult, each couple should be assessed for factors that may optimise or contraindicate the planned pregnancy, possible underlying causes of infertility, and the impact of infertility on the individual and relationship (pg 27).

Grade D, Level 4

D A detailed history-taking and clinical examination should be carried out for couples with fertility concerns (refer to Annex A for details) (pg 27).

Grade D, Level 4

D Patients meeting these criteria should be referred to specialists:

- Women aged $<30$ years who are unable to conceive after regular unprotected intercourse for two years without any known reproductive pathology.

- Women aged $>30$ years who are unable to conceive after regular unprotected intercourse for one year without any known reproductive pathology.

- Patients with a known history of reproductive pathology, e.g. amenorrhoea, pelvic inflammatory diseases, endometriosis.

- Patients with a known history or reason for infertility.

- The presence of male problems e.g. history of urogenital surgery varicocele, significant systemic illness (pg 27).

Grade D, Level 4

B Semen analysis should be conducted as part of initial investigation and should be compared to the following World Health Organization (WHO) Global reference values (fertile men) 2009: 
- Volume (mL): 1.5 or more

- $\mathrm{pH}:>7.2$

- Sperm concentration: 15 million spermatozoa per mL or more

- Total sperm number: 39 million spermatozoa per ejaculate or more

- Motility (PR + NP\%): 40\% or more motile*

- Vitality (\%): 58

- White blood cells $\left(10^{6}\right.$ per $\left.\mathrm{mL}\right):<1.0$

- Morphology (\%): 4 or more

*PR: progressive motility (WHO 1999, grades a + b); NP: non-progressive motility (WHO 1999, grade c) (pg 28).

Grade B, Level 2+

B If the first sperm analysis result is abnormal, the patient should be offered a repeat test from the same laboratory at least three months after the initial analysis (pg 28).

Grade B, Level 2++

Severe abnormality (azoospermia or severe oligozoospermia) of the initial sperm sample, however, warrants an immediate referral to a tertiary centre (see section on male infertility) (pg 28).

D Sperm function tests, screening for antisperm antibodies and postcoital tests on cervical mucus should not be offered, as there is no evidence of effective treatment to improve fertility (pg 28).

Grade D, Level 4

D Women with fertility concerns should have their menstrual history taken (pg 29).

Grade D, Level 4

D Use of basal body temperature charts and home ovulation kits alone to predict ovulation should not be recommended to patients with fertility problems, as these are not always reliable in predicting ovulation, and lead to unnecessary anxiety and stress for the patient (pg 29).

Grade D, Level 4

B Women with infertility should be offered a blood test to measure mid-luteal serum progesterone levels (about seven days before the expected menstrual cycle). If cycles are irregular or prolonged, this test may need to be repeated again weekly thereafter until the next menstrual period (pg 29).

Grade B, Level 2++

B Follicle-stimulating hormone ( $\mathrm{FSH}$ ) and luteinising hormone $(\mathrm{LH})$ investigations should be done on day $2-3$ of the menstrual cycle. Patients with high levels of gonadotrophins should be informed that they are likely to have reduced fertility (pg 29).

Grade B, Level 2+

B Women with infertility should be offered screening for Chlamydia trachomatis before undergoing instrumentation (pg 29).

Grade B, Level 2++

B If screening for Chlamydia trachomatis has not been carried out, prophylactic antibiotics should be given before uterine instrumentation (pg 29).

Grade B, Level 2++

GPP The result of semen analysis and assessment of ovulation should be completed before the clinician embarks on tubal assessment (pg 30).

GPP

B Hysterosalpingography (HSG) should be used as the firstline investigation in tubal assessment (pg 30).

Grade B, Level 2++

A Chlamydia antibody titre (CAT) should be considered as an alternative to HSG if the resources are available (pg 30).

Grade A, Level 1+

B Laparoscopy and dye hydrotubation should be offered to women with comorbidities, such as pelvic inflammatory disease, previous ectopic pregnancy or endometriosis (pg 31).

Grade B, Level 2+

C Fertiloscopy and transvaginal hydrolaparoscopy should not be offered routinely as an alternative to laparoscopy hydrotubation, as their diagnostic accuracy still requires further evaluation (pg 31).

Grade C, Level 3

C When available, transvaginal ultrasound may be used as a screening test for the assessment of uterine cavity in subfertile women (pg 32).

Grade C, Level 2+

B Operative hysteroscopy should not be offered as an initial investigation (pg 32).

Grade B, Level 2++

\section{Ovulatory dysfunction}

C For patients with functional hypothalamic pituitary failure who desire fertility, ovulation induction therapies may be 
indicated. However, the achievement of a healthy weight and modification of lifestyle should be tried first (pg 34).

Grade C, Level 2+

C Where amenorrhoea (which occurs in women with functional hypothalamic pituitary failure) has occurred for longer than a year in duration, assessment of the bone mineral densities should be considered (pg 34).

Grade C, Level 2+

When drug-induced anovulation is suspected, medications should be altered or discontinued, if possible. If the medication which causes anovulation cannot be altered or discontinued, referral to a reproductive medicine specialist for further management is indicated (pg 35).

GPP

GPP Patients with anorexia nervosa should be referred to a mental health care provider for further management (pg 35).

Patients with organic lesions of the hypothalamus and pituitary gland should be referred to a reproductive specialist (pg 35).

C Women should be told that premature ovarian failure is not a definitive diagnosis of infertility, as approximately $5 \%-10 \%$ of these women may conceive spontaneously and unexpectedly after the diagnosis (pg 36).

Grade C Level 2+

C Women with spontaneous premature ovarian failure should be referred to an endocrinologist to investigate asymptomatic autoimmune adrenal insufficiency (pg 36).

Grade C, Level 3

Fertility options in women with premature ovarian failure include the use of an oocyte (egg) donor or embryo donor in an Assisted Reproductive Programme (ARP) (pg 36).

Grade C, Level 2+

A Dopamine receptor agonists are the first-line treatment for patients with idiopathic hyperprolactinaemia secondary to pituitary adenoma (pg 36).

Grade A, Level 1+

C Surgical transsphenoidal resection of microadenomas should not be the primary therapeutic approach for patients with hyperprolactinaemia secondary to pituitary adenoma (pg 37).

Grade C, Level 2+

\section{Managing infertility in polycystic ovary syndrome (PCOS)}

D Diagnosis of polycystic ovary syndrome should only be made when other aetiologies have been excluded (thyroid dysfunction, congenital adrenal hyperplasia, hyperprolactinaemia, androgen secreting tumours and Cushing's syndrome) (pg 38).

Grade D, Level 4

D The following investigations should be done to exclude other aetiologies before a diagnosis of polycystic ovary syndrome is made:

\section{- Baseline laboratory screen:}

- Thyroid function test (thyroid dysfunction can present as amenorrhoea)

- Serum prolactin (hyperprolactinaemia can present as amenorrhoea)

- 17 hydroxyprogesterone (only in the presence of clinical or biochemical evidence of hyperandrogenism; congenital adrenal hyperplasia can present as amenorrhoea and hyperandrogenism)

- Free Androgen Index (FAI = total testosterone divided by sex hormone binding globulin $\times 100$ to give a calculated free testosterone level) or free/bioavailable testosterone (hyperandrogenism as one of the criteria needed to diagnose polycystic ovary syndrome)

- Diagnostic imaging:

Pelvic ultrasound scan to determine features in accordance with the Rotterdam criteria as well as to exclude androgen secreting tumours of the adrenals or ovaries (pg 39-40).

Grade D, Level 4

D The following are optional tests in the diagnosis of polycystic ovary syndrome:

- Gonadotrophin may be required to determine cause of amenorrhoea (primary ovarian failure)

- Fasting insulin is not routinely necessary, but may be considered in those undergoing ovulation induction

- ACTH stimulation test needs to be considered if morning 17 hydroxyprogesterone $>5 \mathrm{nmol} / \mathrm{L}$ in order to exclude non-classical congenital adrenal hyperplasia (pg 40).

Grade D, Level 4

GPP Before any intervention is initiated for women with polycystic ovary syndrome, preconceptional counselling should be provided, emphasising the importance of lifestyle, especially weight reduction and exercise in overweight women, smoking and alcohol consumption (pg 41).

GPP 
A The recommended first-line treatment for ovulation induction remains antiestrogen clomiphene citrate (pg 41).

Grade A, Level 1+

A Patients with polycystic ovary syndrome should be informed that there is an increased risk of multiple pregnancy with ovulation induction using clomiphene citrate (pg 41).

Grade A, Level 1+

GPP Ultrasound monitoring of follicular development at least during the first cycle of treatment with clomiphene is advisable to ensure that the women receive a dose that minimises the risk of multiple pregnancy (pg 41).

GPP

D Recommended second-line intervention for infertility in women with polycystic ovary syndrome is either exogenous gonadotrophins or laparoscopic ovarian surgery (pg 42).

Grade D, Level 4

GPP The use of exogenous gonadotrophins is associated with increased chances of multiple pregnancy, and therefore, intense monitoring of ovarian response is required (pg 42).

GPP

B Laparoscopic ovarian surgery alone is usually effective in $<50 \%$ of women, and additional ovulation induction medication is required under those circumstances (pg 42).

Grade B, Level 2++

B Low-dose therapy with gonadotrophin is recommended, as it offers a significantly lower risk of ovarian hyperstimulation in women with polycystic ovary syndrome (pg 42).

Grade B, Level 2++

C The recommended third-line treatment for infertility in women with polycystic ovary syndrome is in vitro fertilisation (pg 42).

Grade C, Level 2+

GPP As aromatase inhibitors are currently not licensed for use as ovulation induction, we do not recommend the clinical use of aromatase inhibitors for routine ovulation induction (pg 43).

The routine use of metformin in ovulation induction is not recommended as monotherapy or in combination with clomiphene citrate (pg 43).

Grade D, Level 3
B There is currently insufficient evidence to suggest improvement in live birth rates on treatment with metformin before or during assisted reproductive technique cycles, and its routine use is not recommended (pg 43).

Grade B, Level 2+

A The combined use of metformin and clomiphene citrate is recommended for women with clomiphene resistance, especially if they are obese (BMI $\left.>27.5 \mathrm{~kg} / \mathrm{m}^{2}\right)(\mathrm{pg} 44)$.

Grade A, Level 1+

GPP There is insufficient evidence to recommend the widespread use of metformin in pregnant women with polycystic ovary syndrome (pg 45).

GPP

GPP Young women diagnosed with polycystic ovary syndrome should be informed of the possible long-term risks to health that are associated with their condition (pg 45).

GPP

B Patients presenting with polycystic ovary syndrome, particularly if they are obese, have a strong family history of type 2 diabetes, or are over the age of 40 , should be offered a glucose tolerance test (pg 45).

Grade B, Level 1+

B Women diagnosed with polycystic ovary syndrome should be asked (or their partners asked) about snoring and daytime fatigue/somnolence, and be informed of the possible risk of sleep apnoea, and offered investigation and treatment, when necessary (pg 46).

Grade B, Level 2++

B Clinicians should continue to identify cardiovascular risk factors (including blood pressure, cholesterol, triglycerides and high density lipoprotein cholesterol) in women with polycystic ovary syndrome and treat these accordingly (pg 46).

Grade B, Level 2++

A Women diagnosed with polycystic ovary syndrome should be advised regarding weight loss through diet and exercise (pg 47).

Grade A, Level 1+

B Combining metformin and lifestyle modification, including calorie restriction and exercise to facilitate weight loss and attenuate central adiposity, is recommended for obese patients with polycystic ovary syndrome. Higher doses of metformin, up to $2.5 \mathrm{~g} /$ day, may be recommended to achieve an optimal response (pg 47).

Grade B, Level 1+ 
D Ovarian electrocautery should only be reserved for slim women with anovulatory polycystic ovary syndrome (pg 48).

Grade D, Level 3

B Women who have been diagnosed as having polycystic ovary syndrome before pregnancy, especially those requiring ovulation induction for conception, should be screened for gestational diabetes before 20 weeks of gestation, with referral to a specialist obstetric diabetic service if abnormalities are detected (pg 49).

Grade B, Level 1+

\section{Male infertility}

GPP Both male and female partners should be investigated concurrently for couples who present for infertility assessment (pg 50).

B Semen analysis should be done as the primary investigation for male infertility (see section 4) (pg 50).

Grade B, Level 2+

GPP Hormonal investigations for male infertility should be limited to FSH, LH and testosterone levels (pg 50).

D Karyotyping and $\mathrm{Y}$ microdeletion test should be considered for men with nonobstructive azoospermia (pg 51).

Grade D, Level 3

Referral to a tertiary centre should be made for:

- Patients with azoospermia

- Patients with severe semen abnormality

- Patients with clinical evidence of varicocele

- Patients with erectile dysfunction

- Patients with anejaculation

- Patients with retrograde ejaculation

- Patients with suspected androgen deficiency

- Couples who will benefit from assisted reproductive technologies (pg 51).

GPP General advice, such as cessation of smoking, steroid use and withdrawal of offensive medication could be given at primary setting (pg 51).

Couples trying to conceive should generally avoid exposure to harmful chemicals (e.g. by wearing protective clothing when appropriate to reduce the risk of exposure to harmful chemicals) (pg 51).
A Antioxidant supplementation for subfertile males may be used to improve live birth and pregnancy rates for subfertile couples (pg 52).

Grade A, Level 1+

GPP Referral to a fertility specialist should be facilitated if the female is over 35 years old (pg 52).

GPP

B Varicocele treatment may be considered when the female evaluation is normal and the man has a palpable varicocele with suboptimal semen quality (pg 52).

Grade B, Level 2++

GPP In azoospermia, spermatozoa may be retrieved from the testis/epididymis using a variety of techniques such as testicular sperm aspiration (TESA), testicular sperm extraction (TESE), microsurgical epididymal sperm aspiration (MESA) and percutaneous epididymal sperm aspiration (PESA). Sperm cryopreservation should be offered at the time of sperm retrieval (pg 53).

GPP

GPP When retrograde ejaculation is suspected, post-ejaculatory urinalysis (looking for presence of sperm and fructose) may be requested (pg 53).

GPP

C It is important to exclude retrograde ejaculation and anejaculation from other causes of azoospermia, as drug therapy may be used to allow spontaneous conception (pg 53).

Grade C, Level 3

D When available, penile electrovibration and transrectal electroejaculation should be considered before embarking on surgical sperm retrieval and intracytoplasmic sperm injection (pg 54).

Grade D, Level 4

GPP Delayed ejaculation and anorgasmia may have biogenic or psychogenic aetiology. After exclusion of medical illnesses, referral could be made to a sexual therapist who could help in education, counselling and instruction in revised sexual technique to maximise sexual arousal (pg 54).

GPP

B Management of premature ejaculation depends on the underlying aetiology, patient's needs and preference. For lifelong premature ejaculation, selective serotonin reuptake inhibitors (SSRIs) are preferred, while for secondary premature ejaculation, behavioural techniques are the preferred option (pg 54).

Grade B, Level 2++ 
B Patient with premature ejaculation should be informed that daily SSRI is more effective than on-demand SSRI treatment. On-demand use of topical anaesthetics and tramadol may prolong intravaginal ejaculatory latency (pg 54).

Grade B, Level 2++

B Phosphodiesterase type 5 (PDE 5) inhibitors should not be prescribed to men with premature ejaculation when there is no associated erectile dysfunction (pg 54).

Grade B, Level 2++

GPP All patients presenting with erectile dysfunction should have their history taken and assessment done to identify cardiovascular risk factors such as hypertension, hyperdyslipidaemia and diabetes, as these are commonly associated with cardiovascular disease (pg 55).

GPP Although referral to a fertility clinician can help with fertility issues by doing in vitro fertisilisation/intracytoplasmic sperm injection, multidisciplinary referral should be considered in the following situations:

- Complex endocrine disorder (to endocrinologist)

- History of pelvic or perineal trauma, penile deformities or penile implants (to urologist)

- Psychosocial issues or relationship problems (to counsellor, psychologist, psychiatrist) (pg 55).

D First-line treatment for erectile dysfunction should include patient counselling and education, risk factor modification (smoking cessation, reduce alcohol, improved diet and exercise, weight loss) and addressing psychosocial issues (relationship difficulties, anxiety) (pg 55).

Grade D, Level 4

A Oral agents (PDE 5 inhibitors) such as tadalafil (Cialis), sildenafil (Viagra), vardenafil (Levitra) have similar efficacy, tolerability and safety for the treatment of erectile dysfunction. Choice of drug should be individualised based on patient needs (pg 55).

Grade A, Level 1+

D Phosphodiesterase type 5 (PDE 5) inhibitors should not be taken with nitrate-containing medications for erectile dysfunction, as the concurrent use of nitrate medications and PDE 5 inhibitors is contraindicated. Patients need to be educated that they require sexual stimulation for these medications to work (pg 56).

Grade D, Level 4
C Vacuum devices and rings are suitable for men with erectile dysfunction who have contraindications for pharmacologic therapies. It should only be prescribed by clinicians who are familiar with its use (pg 56).

Grade C, Level 2+

C Men with erectile dysfunction who are resistant to PDE 5 inhibitors should be referred to a urologist as combination therapy (PDE 5 inhibitor plus vacuum erectile device, intraurethral medication, intracavernosal injection, androgen supplement, $\alpha$-blocker) or invasive treatment such as penile implant may be considered (pg 56).

Grade C, Level 2+

D Hormone assays should be performed to test for androgen deficiency. As there is diurnal rhythm in hormone secretion, blood samples for testosterone should be taken in the morning (pg 56).

Grade D, Level 4

GPP The result of hormone assays should be interpreted with caution, as there is no appropriate standardised reference range for all laboratories. Therefore, clinical assessment (recent changes in sexual function, patterns of body hair and secondary sexual characteristics) is important to diagnose androgen deficiency (pg 56).

GPP

B When fertility is desired, testosterone should not be used to treat androgen deficiency. When the causes are secondary to hypothalamus or pituitary disorders, human chorionic gonadatropin (HCG) injection may be used instead (pg 57).

Grade B, Level 2++

\section{Tubal-infertility (preventive strategies \& treatment)}

C Women with high risk profiles (early sexual debut, multiple partners, noncompliance with safe sexual advice, etc.) should be screened for Chlamydia trachomatis in their urogenital tracts and be promptly treated to prevent future repercussions, including tubal infertility (pg 58).

Grade C, Level 2+

C Partners of Chlamydia-positive women should be tested and treated to prevent reinfection of the treated women. Positive cases should be referred to the Department of STI Control for contact tracing and treatment (pg 58).

Grade C, Level 2+

A Oral doxycycline (100 mg twice daily for 7-14 days) and azithromycin ( $1 \mathrm{~g}$ stat dose) are recommended antibiotics against Chlamydia trachomatis (pg 58).

Grade A, Level 1++ 
B High-risk women who are scheduled for invasive instrumentation of the reproductive tract should be empirically treated for Chlamydia to prevent ascending infection of the upper reproductive tract, or reactivation of past infection (pg 59).

Grade B, Level 2++

Assessment of tubal patency should be considered in all infertile women (pg 59).

GPP

B Women with low risk for tubal disease (based on the history and physical examination), should be screened with a HSG for tubal patency as part of assessment for infertility (pg 59).

Grade B, Level 2++

\section{Endometriosis}

C A detailed vaginal examination with bimanual palpation and/or rectal examination is essential to detect nodular lesion on the uterosacral ligaments, rectovaginal septum or other surfaces accessible digitally. The detection rate of physical examination is better during menstruation (pg 60).

Grade C, Level 2+
C Magnetic resonance imaging may be considered as an adjunctive investigation tool to laparoscopy in the diagnosis of deeply infiltrating endometriosis (pg 61).

Grade C, Level 2+

B Serum CA125 should not be used in the routine investigation of endometriosis due to inadequate specificity and sensitivity (pg 61).

Grade B, Level 2++

D Diagnosis of endometriosis should be made at laparoscopy unless disease is visible in the vagina or elsewhere (pg 61).

Grade D, Level 4

C Diagnostic laparoscopy for endometriosis should not be undertaken within 3 months of ovarian suppressive treatment, as there is a high risk of missing the lesions and leading to a false negative result (pg 62).

Grade C, Level 2+ 


\section{SINGAPORE MEDICAL COUNCIL CATEGORY 3B CME PROGRAMME} (Code SMJ 201402B)

These questions are based on the full text of the guidelines, which may be found at: http://www.moh.gov.sg/content/moh_web/ healthprofessionalsportal/doctors/guidelines/cpg_medical/2013/cpgmed_infertility.html

Question 1. The following advice could be given to couples to optimise their natural fertility:

(a) Advise couples to time sexual intercourse to coincide with ovulation.

(b) A body mass index of $19-29 \mathrm{~kg} / \mathrm{m}^{2}$ is optimal for women trying to achieve pregnancy.

(c) Smoking is not associated with reduced fertility.

(d) Excessive alcohol intake is detrimental to semen quality.

Question 2. The following should be conducted as part of investigations of infertility:

(a) Semen analysis for men.

(b) Sperm function tests for men.

(c) Taking of menstrual history for women.

(d) Transvaginal hydrolaparoscopy for assessment of tubal damage.

Question 3. In the management of infertility in women with polycystic ovary syndrome:

(a) Preconceptional counselling should be provided to emphasise the importance of lifestyle, especially weight reduction and exercise in overweight women, smoking and alcohol consumption.

(b) The recommended first-line treatment for ovulation induction is the antiestrogen clomiphene citrate.

(c) Metformin should be routinely used in combination with clomiphene citrate for ovulation induction.

(d) Patients presenting with polycystic ovary syndrome, particularly if they are obese, have a strong family history of type 2 diabetes or are over the age of 40 , should be offered a glucose tolerance test.

Question 4. In the management of male infertility:

(a) Semen analysis should be done as the primary investigation.

(b) Advice on cessation of smoking, steroid use and withdrawal of offensive medication should be given at the primary care setting.

(c) PDE 5 inhibitors should be prescribed to men with premature ejaculation, regardless of whether there is associated erectile dysfunction.

(d) PDE 5 inhibitors such as tadalafil, sildenafil and vardenafil have similar efficacy and the choice of drug should be individualised based on patient needs.

Question 5. In the management of tubal infertility:

(a) Women with high risk profiles (e.g. early sexual debut, multiple partners, noncompliance with safe sexual advice) should be screened for Chlamydia trachomatis in their urogenital tracts.

(b) Chlamydia trachomatis should be treated promptly to prevent future repercussions, including tubal infertility.

(c) The assessment of tubal patency should be considered in all infertile women.

(d) A blood test for serum CA125 should be used as routine investigation of endometriosis.

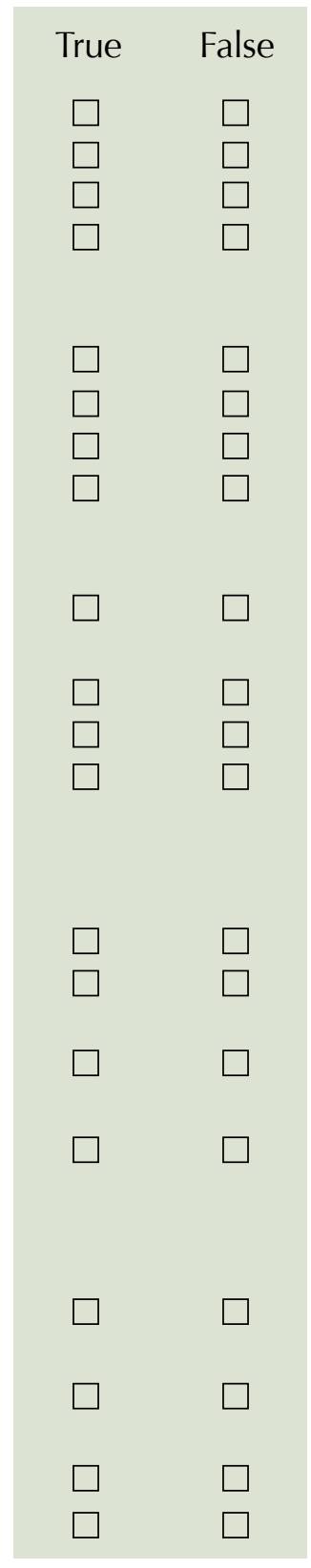

\section{Doctor's particulars:}

Name in full

MCR number

Email address

(1) Log on at the SMJ website: http://www.sma.org.sg/publications/smjcurrentissue.aspx and select the appropriate set of questions. (2) Provide your name, email address and MCR number. (3) Select your answers and click "Submit".

RESULTS:

(1) Answers will be published in the SMJ April 2014 issue. (2) The MCR numbers of successful candidates will be posted online at the SMJ website by 25 March 2014. (3) Passing mark is $60 \%$. No mark will be deducted for incorrect answers. (4) The SMJ editorial office will submit the list of successful candidates to the Singapore Medical Council. (5) One CME point is awarded for successful candidates.

Deadline for submission: (February 2014 SMJ 3B CME programme): 12 noon, 18 March 2014.
} 